\title{
Outcomes and Control Rates for I-125 Plaque Brachytherapy for Uveal Melanoma: A Community-Based Institutional Experience
}

\author{
Aaron Wagner, ${ }^{1}$ Andy Chen, ${ }^{1}$ Taylor Cook, ${ }^{2}$ David Faber, ${ }^{3}$ \\ Kirk Winward, ${ }^{4}$ and William Sause ${ }^{2}$ \\ ${ }^{1}$ Department of Radiation Oncology, Huntsman Cancer Institute, University of Utah, Salt Lake City, UT 84112, USA \\ ${ }^{2}$ Department of Radiation Oncology, Intermountain Medical Center, 5131 Cottonwood Street, Murray, UT 84107, USA \\ ${ }^{3}$ Rocky Mountain Retina Associates, Murray, UT 84107, USA \\ ${ }^{4}$ Retina Associates of Utah, Murray, UT 84107, USA
}

Correspondence should be addressed to William Sause; william.sause@imail.org

Received 31 December 2013; Accepted 11 February 2014; Published 9 March 2014

Academic Editors: M. N. Burnier Jr., M. Nakazawa, and H. Quiroz-Mercado

Copyright (C) 2014 Aaron Wagner et al. This is an open access article distributed under the Creative Commons Attribution License, which permits unrestricted use, distribution, and reproduction in any medium, provided the original work is properly cited.

\begin{abstract}
Purpose. To evaluate our community-based institutional experience with plaque brachytherapy for uveal melanomas with a focus on local control rates, factors impacting disease progression, and dosimetric parameters impacting treatment toxicity. Methods and Materials. Our institution was retrospectively reviewed from 1996 to 2011; all patients who underwent plaque brachytherapy for uveal melanoma were included. Follow-up data were collected regarding local control, distant metastases, and side effects from treatment. Analysis was performed on factors impacting treatment outcomes and treatment toxicity. Results. A total of 107 patients underwent plaque brachytherapy, of which 88 had follow-up data available. Local control at 10 years was $94 \%$. Freedom from progression (FFP) and overall survival at 10 years were $83 \%$ and $79 \%$, respectively. On univariate analysis, there were no tumor or dosimetric treatment characteristics that were found to have a prognostic impact on FFP. Brachytherapy treatment was well tolerated, with clinically useful vision $(>20 / 200)$ maintained in $64 \%$ of patients. Statistically significant dosimetric relationships were established with cataract, glaucoma, and retinopathy development (greatest $P=0.05$ ). Conclusions. Treatment with plaque brachytherapy demonstrates excellent outcomes in a community-based setting. It is well tolerated and should remain a standard of care for COMS medium sized tumors.
\end{abstract}

\section{Introduction}

Uveal melanoma is an uncommon cancer, with age-adjusted incidence rates of 4.3 new cases per million [1]. Mortality however is not rare, with metastases present in up to $20-$ $39 \%$ of patients at 20 years, and tumor related death ranging from $17-20 \%$ at 20 years [2]. There have been multiple investigations into appropriate treatment options, and current accepted standards range from observation to enucleation, all dependent on the size and characteristics of the tumor [3-5].

Brachytherapy is frequently utilized for medium sized tumors (apical height 3-10 $\mathrm{mm}$ and basal diameter 5-16 $\mathrm{mm}$ ) and has been shown to be equivalent to enucleation for tumors in this category [3]. Treatment delivery is nevertheless quite complex, and it has been recommended to only undertake this treatment approach at medical centers with the appropriate expertise [6]. Accordingly, recommendations have been made by the American Brachytherapy Society regarding appropriate treatment delivery and planning [6] .

While the efficacy of plaque brachytherapy has been well established in large institutional practices that are well versed in its implementation [7-9], smaller institutional and community-based results are not as readily available. In addition, while the long term outcomes from plaque brachytherapy have been well established $[3,8-10]$, the literature does not have very well-documented local control rates at long term follow-up [11] to characterize the time at which failures occur. Lastly, comparisons of dosimetric and treatment planning parameters to both treatment toxicity as well as outcomes are not as well recognized, being limited to a select few studies $[8,12-15]$.

The purpose of this study was to evaluate our institutional community-based practice of brachytherapy for uveal melanomas. This included looking at survival rates, local 
control and metastatic rates, and the requirements for salvage treatment. In addition, further investigations were performed to evaluate the factors which predicted progression, as well as dosimetric and tumor factors which predicted ocular complications.

\section{Methods and Materials}

Plaque brachytherapy has been utilized for uveal melanomas at our institution since 1996 and has been performed in a community-based setting. Institutional review board and ethics committee approval was obtained, and patients treated between 1996 and 2011 were retrospectively reviewed, and all patients who underwent plaque brachytherapy for uveal melanoma were included. Data was collected regarding patient demographic data, tumor characteristics, and treatment characteristics. Patients were followed with the treating ophthalmologist, and follow-up data were collected regarding local tumor control, salvage treatment requirements, treatment side effects, occurrence of metastases, and survival status. Tumor characteristics were classified according to the COMS classification, with medium sized tumors having a height of $2.5-10 \mathrm{~mm}$ and diameter of $5-16 \mathrm{~mm}$, large tumors a height of $>10 \mathrm{~mm}$ or diameter $>16 \mathrm{~mm}$, and small tumors heights of $<2.5 \mathrm{~mm}$ and not meeting requirements for medium or large size tumors. AJCC stage was assigned per the AJCC 7th edition staging.

Patients were diagnosed by fundoscopic exam, fluorescein angiography, and B-scan ultrasound examination. Tumor characteristics were recorded including tumor dimensions, location, and distance from intraocular structures.

Plaque implants were performed in accordance with American Brachytherapy Society recommendations [6]. Patients were evaluated by one of three ophthalmologists trained in plaque brachytherapy and our institutional radiation oncology department. Tumors were localized by indirect ophthalmoscopy and occasionally fundoscopic photography in clinic and transillumination at the time of surgery. Plaques of the COMS type were chosen with an additional tumor base margin of at least $2 \mathrm{~mm}$. Treatment was planned in accordance with the American Association of Physicists in Medicine (AAPM) Updated Task Group Number 43 Report. Dose calculations were performed per the COMS protocol using the Bebig Plaque Simulator software. The sources were treated as point sources without corrections for anisotropy, silastic carrier attenuation, scatter from the gold plaque, or L-shell fluorescence. Dose points were carried at the tumor apex, the center of the optic disk, the center of the lens, the scleral surface, and the macula. Iodine-125 (I-125) was utilized in all cases, with a planned dose of $85 \mathrm{~Gy}$ to the tumor apex, or to a $5 \mathrm{~mm}$ depth when tumors were less than $5 \mathrm{~mm}$ in height.

Surgery was performed by the referring ophthalmologist with a radiation oncologist and physicist present. Plaque position was verified either with a dummy plaque or indirect ophthalmoscopy with scleral depression. Muscle transposition was performed if necessitated by tumor position. Plaques were sutured in place and left in place for a time interval as dictated by source strength and desired dose, before being removed.

Planned follow-up was with the treating ophthalmologist, with scheduled appointments planned for 3 to 6 months during the first year, followed by 6 months to annually thereafter. Patients underwent repeat fundoscopic examination, as well as yearly B-scan ultrasound evaluation. Yearly hepatic enzyme evaluation and liver ultrasound as indicated were also performed.

Statistical analysis was performed using StatsDirect statistical software (version 2.7.8, Stats Direct Ltd., Altrincham, UK). Survival was calculated from time of the implant. Kaplan-Meier curves were generated for both overall survival and freedom from progression (OS and FFP, resp.), as well as independently for local control (LC) rates and freedom from distant metastases. FFP was defined as simultaneous local control and the absence of distant metastases. Local control was evaluated by the treating ophthalmologist with repeat fundoscopic examination, and control was defined as no visible tumor growth. If any further salvage treatment was performed, such as laser photocoagulation, this was deemed a failure even in the absence of tumor growth. Univariate analysis was performed by log rank analysis to identify factors impacting FFP. Statistical significance of factors affecting side effect rates was performed with an unpaired $t$-test for continuous variables and Pearson $\chi^{2}$ test for categorical variables. Statistical significance was defined at $P=0.05$.

\section{Results}

Between April of 1996 and November of 2011, 107 patients underwent plaque brachytherapy for uveal melanoma for which dosimetric data were available. Of these, 88 patients had data regarding treatment follow-up. Patient characteristics are displayed in Table 1. For all patients, $85 \%$ of tumors were in the COMS medium sized category, with the majority of the others in the small category. The mean largest diameter was $10.67 \mathrm{~mm}(5.70-18.30 \mathrm{~mm})$, the mean height was $4.49 \mathrm{~mm}(1.60-10.17 \mathrm{~mm})$. The mean distance to the macula was $6.07 \mathrm{~mm}(0-15.73 \mathrm{~mm})$, and the mean distance to the optic disk was $5.93 \mathrm{~mm}(0.01-17.37 \mathrm{~mm})$.

$\mathrm{I}^{125}$ was utilized for all seed implants, and the most common plaque utilized was the COMS 14 size. The average number of seeds was 14 (range 5-24). The average treatment time was 102.11 hours, with an average dose rate of $0.92 \mathrm{~Gy} / \mathrm{hr}$ to the tumor apex. The average seed activity was $3.2 \mathrm{mCi}$. The actual delivered treatment dosimetric characteristics are displayed in Table 2.

Patient OS and FFP is displayed in Figure 1. Median follow-up for the 88 evaluable patients was 48.9 months (range 1-156 months). The five- and ten-year OS for our patient population was $90 \%$ and $79 \%$, respectively (95\% C.I. $79-95 \%$, and $64-88 \%$, resp.). The five- and ten-year FFP was $88 \%$ and $83 \%$, respectively (95\% C.I. $73-95 \%$, and $62-93 \%$, resp.). The local control and metastasis free rates are displayed in Figure 2. Five- and ten-year local control rates were $94 \%$ at both time points (95\% C.I. $82-98 \%$ and $82-98 \%$, resp.), and metastasis free rates were 95 and $89 \%$ at five and ten years 
TABLE 1: Patient and tumor characteristics.

\begin{tabular}{|c|c|c|}
\hline & $\begin{array}{l}\text { All treated } \\
\text { patients }\end{array}$ & $\begin{array}{c}\text { Patients with available } \\
\text { F/U }\end{array}$ \\
\hline Number of Patients & 107 & 88 \\
\hline \multicolumn{3}{|l|}{ Age at diagnosis (yr) } \\
\hline Median & 65 & 65 \\
\hline Range & $22-93$ & $22-93$ \\
\hline \multicolumn{3}{|l|}{ Sex } \\
\hline Male & $60(56 \%)$ & $48(55 \%)$ \\
\hline Female & $47(44 \%)$ & $40(45 \%)$ \\
\hline \multicolumn{3}{|l|}{ Laterality } \\
\hline Left & $56(52 \%)$ & $47(53 \%)$ \\
\hline Right & $51(48 \%)$ & $41(47 \%)$ \\
\hline \multicolumn{3}{|c|}{ Preexisting Conditions } \\
\hline Hypertension & $50(47 \%)$ & $40(45 \%)$ \\
\hline CVS Disease & $16(15 \%)$ & $12(14 \%)$ \\
\hline Diabetes & $15(14 \%)$ & $10(11 \%)$ \\
\hline Smoking History & $17(16 \%)$ & $18(20 \%)$ \\
\hline \multicolumn{3}{|l|}{ COMS Classification } \\
\hline Small & $13(12 \%)$ & $12(14 \%)$ \\
\hline Medium & $91(85 \%)$ & $74(84 \%)$ \\
\hline Large & $3(3 \%)$ & $2(2 \%)$ \\
\hline \multicolumn{3}{|l|}{ Stage } \\
\hline Tla & $39(36 \%)$ & $32(36 \%)$ \\
\hline $\mathrm{T} 1 \mathrm{~b}$ & $1(1 \%)$ & $1(1 \%)$ \\
\hline $\mathrm{T} 2 \mathrm{a}$ & $43(40 \%)$ & $38(43 \%)$ \\
\hline $\mathrm{T} 2 \mathrm{~b}$ & $5(5 \%)$ & $5(6 \%)$ \\
\hline T3a & $15(14 \%)$ & $9(10 \%)$ \\
\hline $\mathrm{T} 3 \mathrm{~b}$ & $3(3 \%)$ & $3(3 \%)$ \\
\hline $\mathrm{T} 4 \mathrm{a}$ & $1(1 \%)$ & $0(0 \%)$ \\
\hline $\mathrm{T} 4 \mathrm{~b}$ & $0(0 \%)$ & $0(0 \%)$ \\
\hline \multicolumn{3}{|l|}{ Equatorial position } \\
\hline Anterior & $8(7 \%)$ & $7(8 \%)$ \\
\hline Posterior & $70(65 \%)$ & $58(66 \%)$ \\
\hline Spanning & $25(23 \%)$ & $20(23 \%)$ \\
\hline Unknown & $4(4 \%)$ & $3(3 \%)$ \\
\hline
\end{tabular}

F/U: follow-up; COMS: collaborative ocular melanoma study; CVS: cardiovascular.

TABLE 2: Delivered dosimetric parameters.

\begin{tabular}{lcc}
\hline & Median dose (Gy) & Range (Gy) \\
\hline Tumor apex & 85.90 & $83.72-147.20$ \\
Inner sclera & 248.95 & $99.99-1132.30$ \\
Opposite retina & 6.44 & $2.84-23.30$ \\
Macula & 46.51 & $8.17-174.47$ \\
Optic disc & 39.78 & $8.18-238.70$ \\
Lens center & 13.31 & $4.53-112.10$ \\
\hline
\end{tabular}

Gy: gray.

(95\% C.I. 79-99\% and 67-97\%, resp.). Local failure was seen in only three patients, one of whom underwent enucleation, and two patients underwent transpupillary thermotherapy. Fifteen patients also underwent laser photocoagulation at the time of plaque implant.

A univariate analysis was then performed for factors affecting FFP. Factors evaluated included patient age, sex, dose to the tumor apex, and tumor characteristics including COMS classification, AJCC stage, tumor location by equatorial position, involvement of the ciliary body, tumor height, and plaque margin on the tumor. Dose was dichotomized both to doses to the apex of $<$ or $\geq 85$ Gy as well as $<$ or $\geq 90 \mathrm{~Gy}$. Results are displayed in Table 3 . There were no statistically significant variables impacting FFP seen. With no significant variables on univariate analysis, a multivariate analysis was not performed.

Overall, treatment was well tolerated. Cataract formation and radiation retinopathy were seen in $25 \%$ and $22 \%$ of patients, respectively. Glaucoma was noted after treatment in $6 \%$ of patients. Optic neuropathy was seen in 3\% of patients. Other complications noted included mild/transient diplopia, retinal detachment/hemorrhage, and conjunctival irritation. Decreased visual acuity was the most common functional change seen, with $64 \%$ of patients maintaining best corrected vision of 20/200 or better. Results are displayed in Table 4.

The impact of dosimetric variables and select tumor characteristics on treatment side effects was then investigated. Variables were chosen in accordance to logical associations, and a blanket analysis of all known factors was not performed. A statistically significant correlation between lens dose and cataract formation was seen $(P=0.05)$. In addition, correlations between lens dose and glaucoma formation, as well as both tumor apex and $5 \mathrm{~mm}$ depth dose to radiation retinopathy, were also noted $(P=0.04, P=0.01$, and $P=$ 0.04 , resp.). No other dosimetric variables were statistically noted to have an impact on the incidence of side effects to treatment. Results are displayed in Table 5.

\section{Discussion}

The results of the COMS $[3,16]$ studies have well demonstrated that plaque brachytherapy is an acceptable treatment modality for medium sized tumors. The 5 -year OS was $82 \%$ and $81 \%$, and rates of distant metastases $91 \%$ and $89 \%$ for the patients receiving plaque brachytherapy and enucleation, respectively, with no statistical difference between treatment modality [16]. Longer term follow-up demonstrated that there continued to be no survival difference between these two treatment modalities [3], and in light of lower treatment morbidity plaque brachytherapy has become the predominant treatment modality [17].

While the COMS study has validated the implementation of plaque brachytherapy for medium sized tumors, the process is complex and special considerations must be undertaken in a multidisciplinary approach [6]. As such it is important to verify that the results obtained by the COMS study can be translated into clinical practice and that results are not limited to a few large institutions. Many of the largest studies to date have come from select institutions 


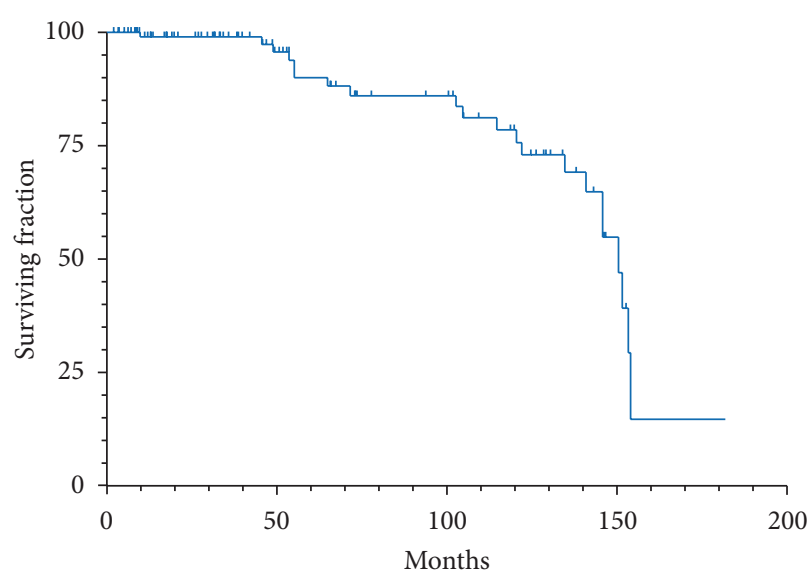

(a)

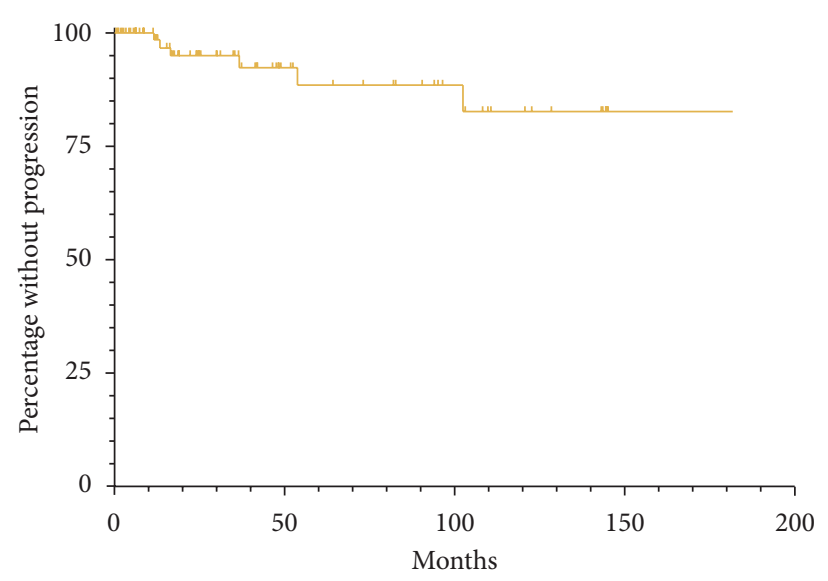

(b)

FIGURE 1: (a) Overall survival of patient population. (b) Freedom from progression from either local disease or distant metastases.

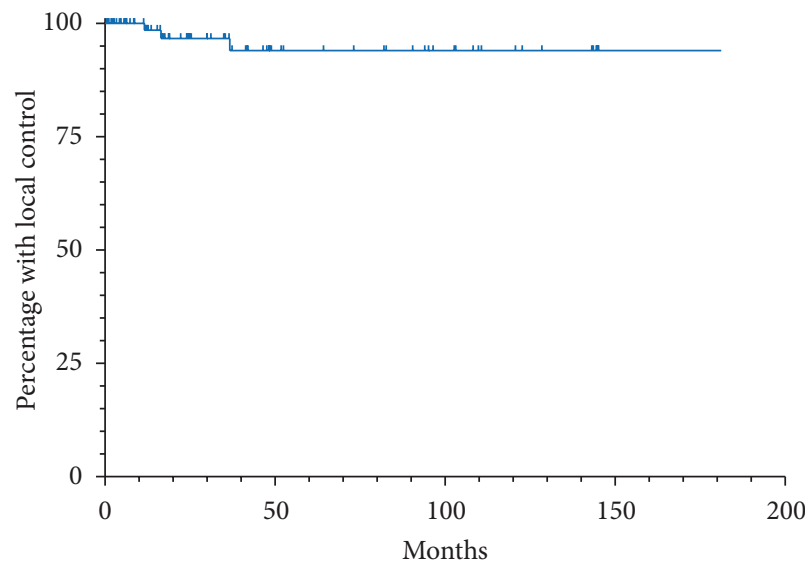

(a)

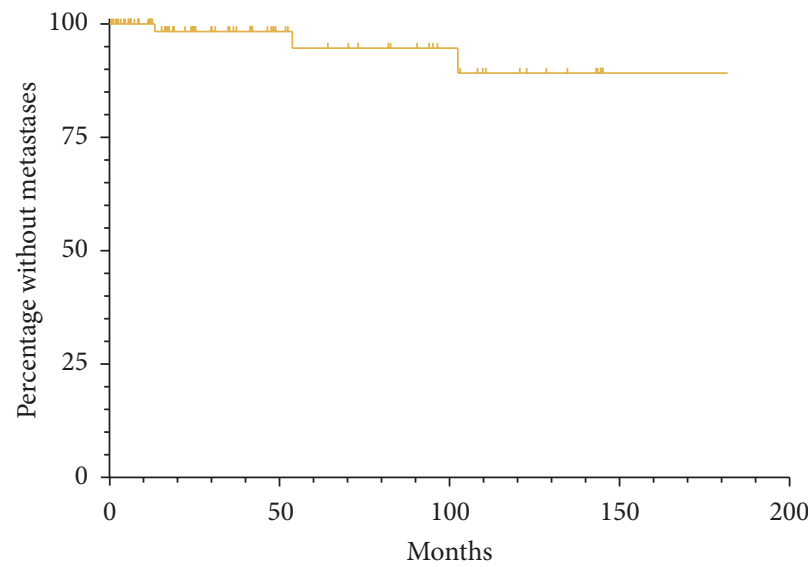

(b)

FIgURE 2: (a) Local control. (b) Freedom from metastatic disease.

[7-10, 15, 18-21], and reports from other clinical practices are much more limited.

The results of our study also demonstrate excellent outcomes for the utilization of plaque brachytherapy. Our 5and 10 -year OS rates were 90 and 79\%, respectively. At the same time intervals, $88 \%$ and $83 \%$ remained free from either local failures or distant metastases. These results are similar to the COMS report [3], where 10-year OS for both groups cumulatively was $65 \%$, and 10 -year survival in the absence of metastatic disease was $83 \%$, demonstrating that at least equivalent results can be obtained in a community practice based setting.

Our results demonstrated local control which plateaued after 3 years, with long term local control being 94\%. These results compare favorable to the COMS study, where 5year local control rates were reported as $89.7 \%$ [22]. Several institutions have shown similar control rates, ranging from 81 to $93 \%[12,15,21,23]$, and, in a study by Leonard et al. [11], a literature review was performed where the 5-year local control average was thought to be $88.1 \%$ for I-125 plaques. However, it was noted in the same study that long term local control has not been as well established, with rates confined almost entirely to select few studies [7, 18, 19, 24]. While a limited number of patients were evaluable for long term follow-up in our study (for LC only 11 patients at 10 years), the 10-year local control rate was $94 \%$, with only 3 patients having experienced a true local failure. In addition, these failures all occurred within the first 3 years of therapy. Nevertheless, other studies have continued to show late recurrences after 5 years [18], and our study also demonstrated an even distribution of metastases over the time period evaluated, indicating the need for continued follow-up and close monitoring long term.

In our study, no significant prognostic factors were seen to impact FFP rates. This includes patient demographic variables, tumor characteristics, plaque margin, and tumor apex dose. In contrast, in a report by Leonard et al. [11], multiple factors including gender and apical height were predictive of local failure on univariate analysis. Similarly, in a report by Jensen et al. [12], tumor diameter, tumor height, and 
TABLE 3: Univariate analysis on factors impacting.

\begin{tabular}{|c|c|c|}
\hline & HR & 95\% C.I. \\
\hline \multicolumn{3}{|l|}{ Age } \\
\hline$<60$ & Referent & \\
\hline$\geq 60$ & 2.56 & $0.58-11.26$ \\
\hline \multicolumn{3}{|l|}{ Sex } \\
\hline Male & Referent & \\
\hline Female & 2.00 & $0.44-9.06$ \\
\hline \multicolumn{3}{|c|}{ COMS classification } \\
\hline Small & 0.84 & $0.11-6.13$ \\
\hline Medium/large & Referent & \\
\hline \multicolumn{3}{|l|}{ T-stage } \\
\hline 1 & 0.68 & $0.13-3.69$ \\
\hline 2 & Referent & \\
\hline 3 & 1.11 & $0.16-7.75$ \\
\hline 4 & 2.71 & $0.16-7.73$ \\
\hline \multicolumn{3}{|l|}{ Equatorial position } \\
\hline Anterior & 1.07 & $0.21-5.46$ \\
\hline Spanning & 1.66 & $0.11-25.64$ \\
\hline Posterior & Referent & \\
\hline \multicolumn{3}{|c|}{ Ciliary body involvement } \\
\hline Not involved & Referent & \\
\hline Involved & 1.89 & $0.12-28.75$ \\
\hline \multicolumn{3}{|l|}{ Tumor height } \\
\hline$<5 \mathrm{~mm}$ & Referent & \\
\hline$\geq 5 \mathrm{~mm}$ & 1.04 & $0.23-4.67$ \\
\hline \multicolumn{3}{|l|}{ Plaque margin } \\
\hline$<3.5 \mathrm{~mm}$ & 2.79 & $26.08-0.30$ \\
\hline$\geq 3.5 \mathrm{~mm}$ & Referent & \\
\hline \multicolumn{3}{|l|}{ Apex dose } \\
\hline$<85 \mathrm{~Gy}$ & 0.33 & $0.03-3.32$ \\
\hline$\geq 85 \mathrm{~Gy}$ & Referent & \\
\hline$<90 \mathrm{~Gy}$ & Referent & \\
\hline$\geq 90 \mathrm{~Gy}$ & 2.13 & $0.46-9.76$ \\
\hline
\end{tabular}

FFP: freedom from progression; COMS: collaborative ocular melanoma study.

TABLE 4: Side effects from treatment.

\begin{tabular}{lc}
\hline Side effects & \\
\hline Pts with evaluable f/u & 88 \\
Clinically useful vision & $56(64 \%)$ \\
Cataracts & $21(24 \%)$ \\
Radiation retinopathy & $19(22 \%)$ \\
Optic neuropathy & $3(3 \%)$ \\
Glaucoma & $5(6 \%)$ \\
\hline
\end{tabular}

tumor apex dose, as well as other factors, were predictive of either local failure or distant metastasis. In the COMS study [3], tumor diameter, apical height, and equatorial position were all predictive of development of distant metastasis. While these results certainly contrast with our study, the lack of prognostic impact can be explained fairly readily by the low instances of either local failures or distant metastases,
TABLE 5: Analysis of factors predicting for treatment side effects.

\begin{tabular}{lccc}
\hline & $\begin{array}{c}\text { Absent } \\
\text { (mean) }\end{array}$ & $\begin{array}{c}\text { Present } \\
\text { (mean) }\end{array}$ & $P$ value \\
\hline Cataracts & & & \\
$\quad$ Lens dose (Gy) & 14.67 & 24.64 & 0.05 \\
Glaucoma & 17.12 & 32.11 & 0.04 \\
$\quad$ Lens dose (Gy) & & & \\
Retinopathy & 78.48 & 108.6 & 0.01 \\
5 mm depth dose (Gy) & 280.65 & 333 & 0.14 \\
Inner dclera dose (Gy) & 10.47 & 10.52 & 0.93 \\
Tumor size (mm) & 4.26 & 4.68 & 0.40 \\
Tumor height (mm) & 91.19 & 96.99 & 0.04 \\
Dose apex (Gy) & & & \\
Optic neuropathy & 62.51 & 95.7 & 0.22 \\
Macula dose & 47.06 & 48.83 & 0.93 \\
Optic disk dose & & & \\
Clinically useful vision & 11.22 & 10.28 & 0.10 \\
Tumor size (mm) & 98.34 & 83.48 & 0.15 \\
5 mm depth dose (Gy) & 55.25 & 44.81 & 0.25 \\
Optic disk dose (Gy) & 343.67 & 280.75 & 0.10 \\
Inner sclera dose (Gy) & 75.49 & 60.31 & 0.20 \\
Macula dose (Gy) & & & \\
\hline
\end{tabular}

and these numbers were likely not adequate to demonstrate any statistical significance regarding these outcomes in the absence of higher patient numbers.

The availability of studies evaluating a dosimetric impact on treatment toxicity is relatively limited. Jensen et al. [12] performed evaluations regarding both total doses and dose rates on both treatment outcomes and toxicity. They found associations in visual acuity or blindness with total doses and dose rates to the macula, optic disk, and lens. Gündüz et al. [8] on univariate analysis found maculopathy to be associated with apex dose rate, and papillopathy to be associated with apex dose rate and optic disk dose. Jones et al. [13] noted association between dose rates to the macula and visual decline after treatment. Stack et al. [14] noted relationships between dose and development of maculopathy and cataract formation.

The results of our study also demonstrated causal relationships between total dose to ocular structures and treatment toxicity. A statistical increase in cataract formation was noted with increased mean lens dose $(P=0.05)$. This is not surprising, as the association between lens dose and cataract formation is well known $[25,26]$. In addition, a relationship between lens dose and glaucoma formation was also noted $(P=0.04)$. While the rates of radiation induced glaucoma in our study were relatively low, $6 \%$, neovascularization was noted in almost $50 \%$ of the cases, and a causal relationship of neovascularization caused by radiation induced ischemia, and subsequent neovascular glaucoma has been noted [27]. Lastly, an association between both $5 \mathrm{~mm}$ depth dose and tumor apex dose to retinopathy was also observed $(P=0.01$ and $P=0.04$, resp.), 
indicating a correlation between increased prescription dose and the development of retinopathy. Overall, these results are consistent with institutional reports of the dose dependence of treatment complications, and while the exact relationships vary to some degree between our study and other published reports, it does seem to indicate that causal relationships do exist.

At the same time, while dose-dependent toxicities may be established, this should not necessarily change clinical practice. Tumor control is still the primary objective, and a reduced tumor dose or dose rate to improve toxicity might result in poor control. While the results of our study do not demonstrate any impact on FFP with dose thresholds of $85 \mathrm{~Gy}$ or $90 \mathrm{~Gy}$, other dose effects have been noted. In the aforementioned study by Jensen et al. [12], decreased total dose to the tumor apex of $<100 \mathrm{~Gy}$ and dose rates of $<90 \mathrm{cGy} / \mathrm{hr}$, while both associated with improved toxicity, were also associated with increased rates of metastases, and other studies have also demonstrated similar associations $[8,15]$. While the actual dose and rate thresholds vary in the literature, a threshold does seem to exist. As a result, while dose-dependent toxicity relationships are present, clinical benefits are likely in the realm of improved patient counseling rather than tailored treatment.

Limitations to our study certainly exist. Not all patients had follow-up data available, especially with longer followup approaching 10 years, as indicated by the lower number of patients for whom primary outcomes and treatment complications were evaluable. In addition, with low rates of recurrences and metastases, many more patients would be required to fully establish relationships between dosimetric and tumor characteristics and treatment outcomes. This same critique applies to evaluating causal factors for treatment complications. In addition, it is a retrospective study, and limitations such as loss to follow-up, completeness of treatment records, record uniformity, and variations in follow-up monitoring all apply. Nevertheless, it still serves as a valuable marker of clinical practice in the modern era.

In conclusion, this series continues to demonstrate excellent and reproducible clinical outcomes for plaque brachytherapy for uveal melanomas, with satisfactory long term control. Local recurrences are quite rare, and treatment is well tolerated with decreases in visual acuity being the most common effect of treatment. Relationships appear to exist between treatment toxicity and doses to intraocular structures. Further evaluation would be warranted to fully evaluate these relationships.

\section{Conflict of Interests}

The authors declare that there is no conflict of interests regarding the publication of this paper.

\section{References}

[1] A. D. Singh, L. Bergman, and S. Seregard, "Uveal melanoma: epidemiologic aspects," Ophthalmology Clinics of North America, vol. 18, no. 1, pp. 75-84, 2005.
[2] C. L. Shields, S. Kaliki, M. Furuta, A. Mashayekhi, and J. A. Shields, "Clinical spectrum and prognosis of uveal melanoma based on age at presentation in 8,033 cases," Retina, vol. 32, pp. 1363-1372, 2012.

[3] Collaborative Ocular Melanoma Study Group, "The COMS randomized trial of iodine 125 brachytherapy for choroidal melanoma: V. Twelve-year mortality rates and prognostic factors: COMS report no. 28," Archives of Ophthalmology, vol. 124, no. 12, pp. 1684-1693, 2006.

[4] B. S. Hawkins and Collaborative Ocular Melanoma Study Group, "The Collaborative Ocular Melanoma Study (COMS) randomized trial of pre-enucleation radiation of large choroidal melanoma: IV. Ten-year mortality findings and prognostic factors. COMS report number 24," American Journal of Ophthalmology, vol. 138, pp. 936-951, 2004.

[5] J.-P. Caujolle, H. Mammar, E. Chamorey, F. Pinon, J. Herault, and P. Gastaud, "Proton beam radiotherapy for uveal melanomas at nice teaching hospital: 16 years' experience," International Journal of Radiation Oncology Biology Physics, vol. 78, no. 1, pp. 98-103, 2010.

[6] S. Nag, J. M. Quivey, J. D. Earle, D. Followill, J. Fontanesi, and P. T. Finger, "The American Brachytherapy Society recommendations for brachytherapy of uveal melanomas," International Journal of Radiation Oncology Biology Physics, vol. 56, no. 2, pp. 544-555, 2003.

[7] P. de Potter, C. L. Shields, J. A. Shields, J. R. Cater, and L. W. Brady, "Plaque radiotherapy for juxtapapillary choroidal melanoma: visual acuity and survival outcome," Archives of Ophthalmology, vol. 114, no. 11, pp. 1357-1365, 1996.

[8] K. Gündüz, C. L. Shields, J. A. Shields, J. Cater, J. E. Freire, and L. V. Brady, "Radiation retinopathy following plaque radiotherapy for posterior uveal melanoma," Archives of Ophthalmology, vol. 117, no. 5, pp. 609-614, 1999.

[9] J. M. Quivey, D. H. Char, T. L. Phillips, K. A. Weaver, J. R. Castro, and S. M. Kroll, "High intensity 125-iodine (125I) plaque treatment of uveal melanoma," International Journal of Radiation Oncology Biology Physics, vol. 26, no. 4, pp. 613-618, 1993.

[10] C. L. Shields, J. A. Shields, J. Cater et al., "Plaque radiotherapy for uveal melanoma: long-term visual outcome in 1106 consecutive patients," Archives of Ophthalmology, vol. 118, no. 9, pp. 1219$1228,2000$.

[11] K. L. Leonard, N. L. Gagne, J. E. Mignano, J. S. Duker, E. A. Bannon, and M. J. Rivard, "A 17-year retrospective study of institutional results for eye plaque brachytherapy of uveal melanoma using 125I, 103Pd, and 131Cs and historical perspective," Brachytherapy, vol. 10, no. 4, pp. 331-339, 2011.

[12] A. W. Jensen, I. A. Petersen, R. W. Kline, S. L. Stafford, P. J. Schomberg, and D. M. Robertson, "Radiation complications and tumor control after 125I plaque brachytherapy for ocular melanoma," International Journal of Radiation Oncology Biology Physics, vol. 63, no. 1, pp. 101-108, 2005.

[13] R. Jones, E. Gore, W. Mieler et al., "Posttreatment visual acuity in patients treated with episcleral plaque therapy for choroidal melanomas: dose and dose rate effects," International Journal of Radiation Oncology Biology Physics, vol. 52, no. 4, pp. 989-995, 2002.

[14] R. Stack, M. Elder, A. Abdelaal, R. Hidajat, and R. Clemett, "New Zealand experience I125 brachytherapy for choroidal melanoma," Clinical and Experimental Ophthalmology, vol. 33, no. 5, pp. 490-494, 2005. 
[15] J. M. Quivey, J. Augsburger, L. Snelling, and L. W. Brady, “125I plaque therapy for uveal melanoma: analysis of the impact of time and dose factors on local control," Cancer, vol. 77, no. 11, pp. 2356-2362, 1996.

[16] M. Diener-West, J. D. Earle, S. L. Fine et al., "The COMS randomized trial of iodine 125 brachytherapy for choroidal melanoma, III: initial mortality findings: COMS report no. 18," Archives of Ophthalmology, vol. 119, no. 7, pp. 969-982, 2001.

[17] A. D. Singh, M. E. Turell, and A. K. Topham, "Uveal melanoma: trends in incidence, treatment, and survival," Ophthalmology, vol. 118, no. 9, pp. 1881-1885, 2011.

[18] D. H. Char, S. Kroll, T. L. Phillips, and J. M. Quivey, "Late radiation failures after iodine 125 brachytherapy for uveal melanoma compared with charged-particle (proton or helium ion) therapy," Ophthalmology, vol. 109, no. 10, pp. 1850-1854, 2002.

[19] C. L. Shields, M. Naseripour, J. Cater et al., "Plaque radiotherapy for large posterior uveal melanomas ( $\geq 8$-mm thick) in 354 consecutive patients," Ophthalmology, vol. 109, no. 10, pp. 18381849, 2002.

[20] S. Packer, M. Rotman, and P. Salanitro, "Iodine-125 irradiation of choroidal melanoma. Clinical experience," Ophthalmology, vol. 91, no. 12, pp. 1700-1708, 1984.

[21] S. Packer, S. Stoller, M. L. Lesser, F. S. Mandel, P. T. Finger, and D. M. Robertson, "Long-term results of iodine 125 irradiation of uveal melanoma," Ophthalmology, vol. 99, no. 5, pp. 767-774, 1992.

[22] L. M. Jampol, C. S. Moy, T. G. Murray et al., "The COMS randomized trial of iodine 125 brachytherapy for choroidal melanoma: IV. Local treatment failure and enucleation in the first 5 years after brachytherapy. COMS report no. 19," Ophthalmology, vol. 109, pp. 2197-2206, 2002.

[23] S. Nag, D. Wang, H. Wu, C. J. Bauer, R. B. Chambers, and F. H. Davidorf, "Custom-made "Nag" eye plaques for $125 \mathrm{I}$ brachytherapy," International Journal of Radiation Oncology Biology Physics, vol. 56, no. 5, pp. 1373-1380, 2003.

[24] M. S. Sagoo, C. L. Shields, A. Mashayekhi et al., "Plaque radiotherapy for juxtapapillary choroidal melanoma: tumor control in 650 consecutive cases," Ophthalmology, vol. 118, no. 2, pp. 402-407, 2011.

[25] J. M. Henk, R. A. F. Whitelocke, A. P. Warrington, and E. M. Bessell, "Radiation dose to the lens and cataract formation," International Journal of Radiation Oncology Biology Physics, vol. 25, no. 5, pp. 815-820, 1993.

[26] B. Emami, J. Lyman, A. Brown et al., "Tolerance of normal tissue to therapeutic irradiation," International Journal of Radiation Oncology Biology Physics, vol. 21, no. 1, pp. 109-122, 1991.

[27] J. C. Wen, S. C. Oliver, and T. A. McCannel, "Ocular complications following I-125 brachytherapy for choroidal melanoma," Eye, vol. 23, no. 6, pp. 1254-1268, 2009. 


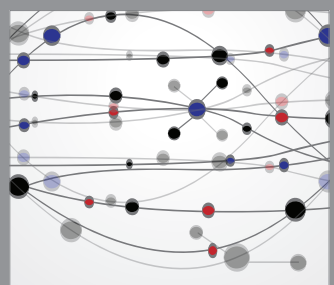

The Scientific World Journal
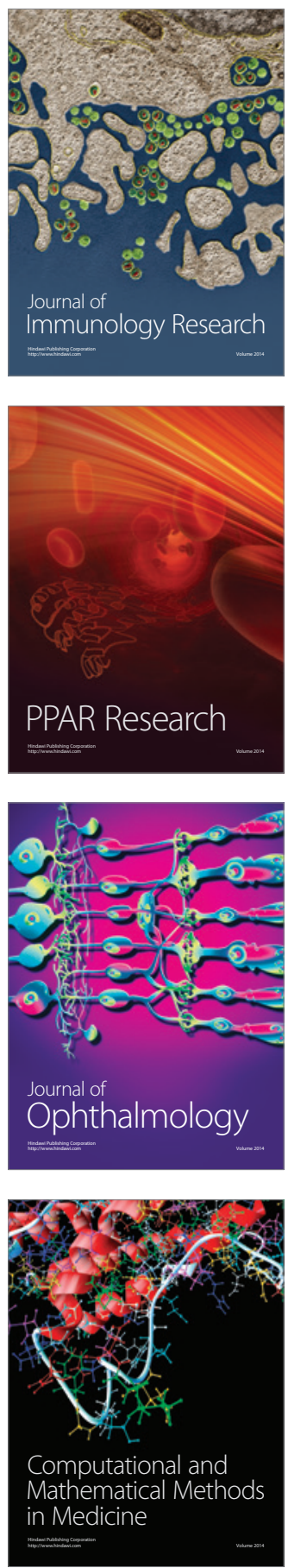

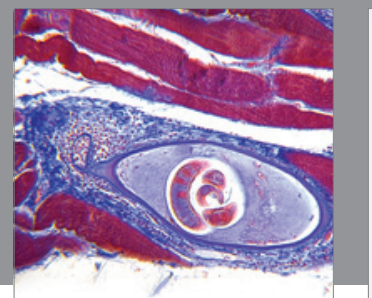

Gastroenterology

Research and Practice
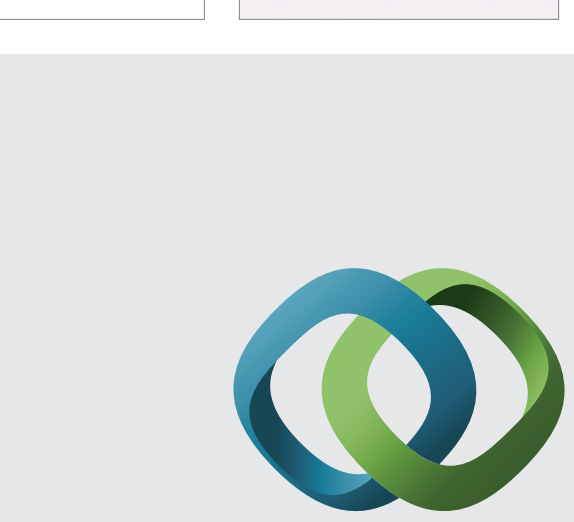

\section{Hindawi}

Submit your manuscripts at

http://www.hindawi.com
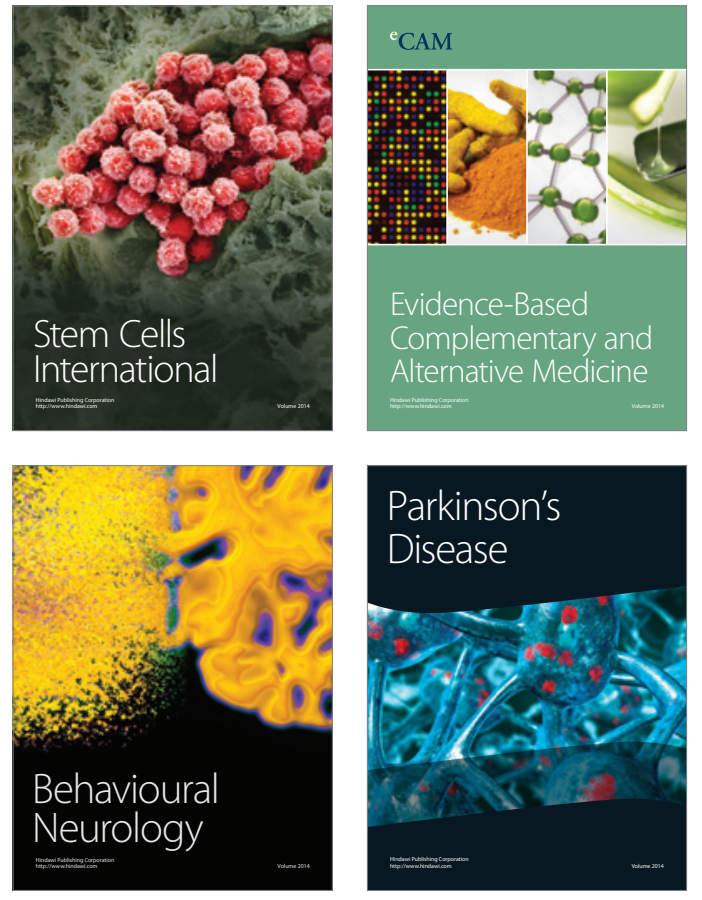
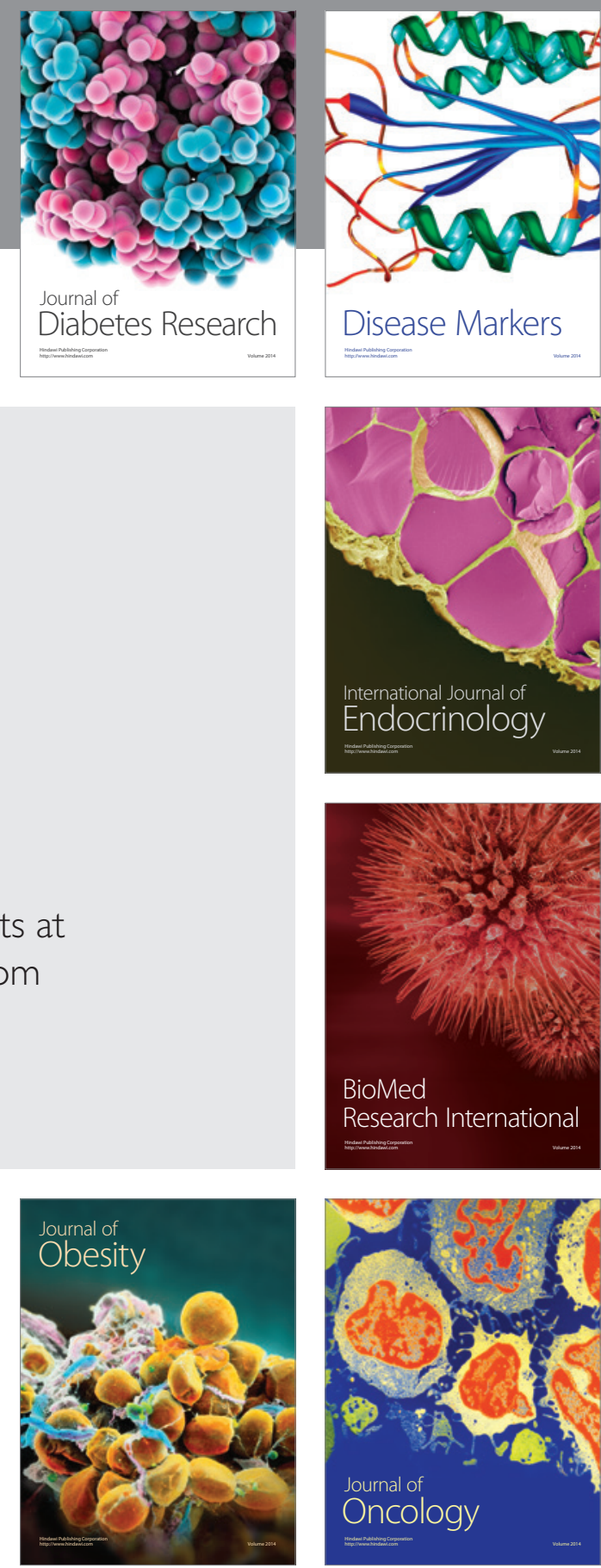

Disease Markers
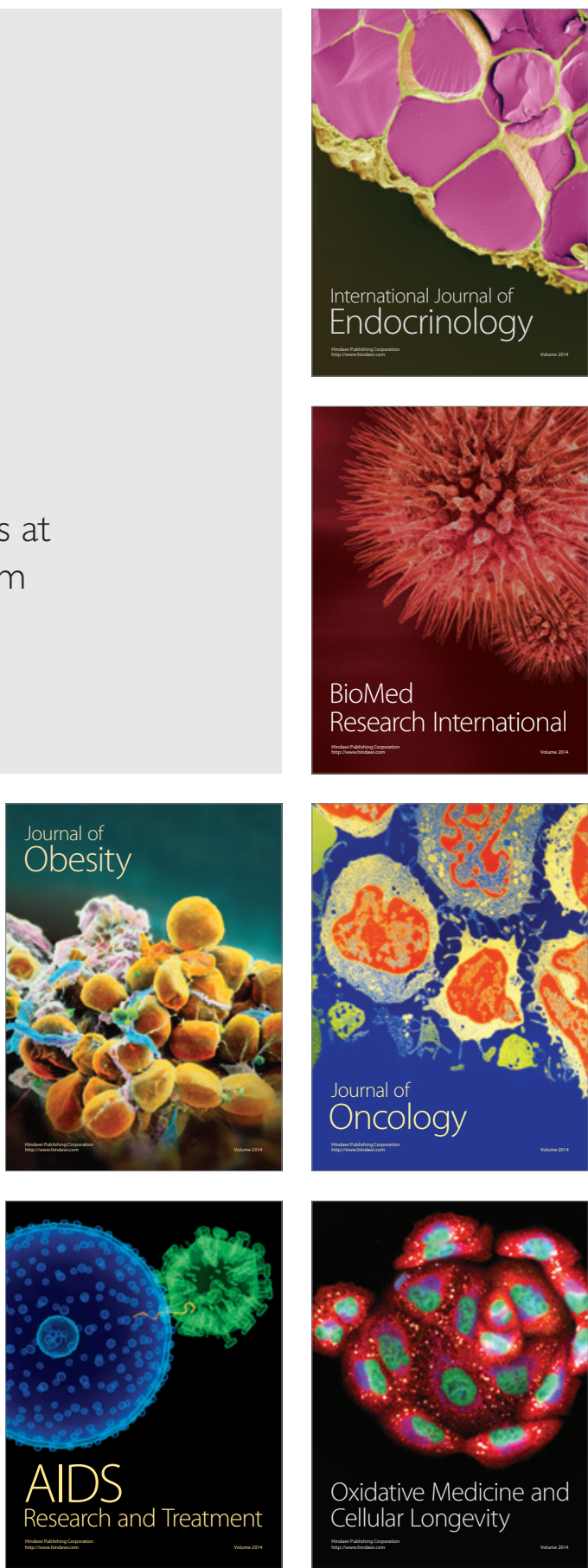